\title{
Policy-Based Computing: From Systems and Applications to Theory
}

\author{
Jorge Lobo \\ IBM T. J. Watson Research Center \\ jlobo@us.ibm.com
}

\begin{abstract}
The need for a more autonomous management of distributed systems and networks has driven research and industry to look for management frameworks that go beyond the direct manipulation of network devices and systems. One approach towards this aim is to build policy-based management systems. Policy-based computing refers to a software paradigm developed around the concept of building autonomous systems that provide system administrators and decision makers with interfaces that let them set general guiding principles and policies to govern the behavior and interactions of the managed systems. Although many of the tasks are still carried out manually and ad hoc, instances of limited policy-based systems can be found in areas such as Internet service management, privacy, security and access management, management of quality of service and service level agreements in networks.

Policies can be specified at many levels of abstraction, from natural language specifications to more elementary condition-action rule specifications. From these specifications policy systems need to come up with implementations. Some of these implementations can be done automatically, others require manual steps. In some cases policies impose legal commitments and systems should be able to demonstrate compliance. There are also situations in which policies are in conflict with each other and a system cannot implement them simultaneously without providing methods for conflict resolution. In this presentation I will review a few policy systems, applications and specification languages. Then I will provide a more formal characterization of policies and their computational model. I will show a simple policy language in the style of the action description language $\mathcal{A}$. I will discuss current solutions to policy conflicts, discuss the problem of policy refinement, i.e. transformations from high level specifications to lower level specifications, current approaches to refinement and provide a partial formalization of the general problem. I will discuss limitations of current systems and directions of research.
\end{abstract}

